\title{
Arginase promotes skeletal muscle arteriolar endothelial dysfunction in diabetic rats
}

\section{Fruzsina K. Johnson ${ }^{1}$, Robert A. Johnson ${ }^{1}$, Kelly J. Peyton ${ }^{2}$, Ahmad R. Shebib $^{2}$ and William Durante ${ }^{2}$}

1 Department of Physiology and Pharmacology, Lincoln Memorial University, Harrogate, TN, USA

${ }^{2}$ Department of Medical Pharmacology and Physiology, University of Missouri, Columbia, MO, USA

\section{Edited by:}

Rudolf Lucas, Medical College of Georgia, USA

\section{Reviewed by:}

Rudolf Lucas, Medical College of Georgia, USA

Ciprian B. Anea, Medical University of

Vienna, Austria

\section{*Correspondence:}

William Durante, Department of

Medical Pharmacology and

Physiology, School of Medicine,

University of Missouri, M409 Medical

Sciences Building, One Hospital

Drive, Columbia, MO 65212, USA.

e-mail:durantew@health.

missouriedu
Endothelial dysfunction is a characteristic feature in diabetes that contributes to the development of vascular disease. Recently, arginase has been implicated in triggering endothelial dysfunction in diabetic patients and animals by competing with endothelial nitric oxide synthase for substrate L-arginine. While most studies have focused on the coronary circulation and large conduit blood vessels, the role of arginase in mediating diabetic endothelial dysfunction in other vascular beds has not been fully investigated. In the present study, we determined whether arginase contributes to endothelial dysfunction in skeletal muscle arterioles of diabetic rats. Diabetes was induced in male Sprague Dawley rats by streptozotocin injection. Four weeks after streptozotocin administration, blood glucose, glycated hemoglobin, and vascular arginase activity were significantly increased. In addition, a significant increase in arginase I and II mRNA expression was detected in gracilis muscle arterioles of diabetic rats compared to age-matched, vehicle control animals. To examine endothelial function, first-order gracilis muscle arterioles were isolated, cannulated in a pressure myograph system, exposed to graded levels of luminal flow, and internal vessel diameter measured. Increases in luminal flow $(0-50 \mu \mathrm{L} / \mathrm{min})$ caused progressive vasodilation in arterioles isolated from control, normoglycemic animals. However, flowinduced vasodilation was absent in arterioles obtained from streptozotocin-treated rats. Acute in vitro pretreatment of blood vessels with the arginase inhibitors $N^{\omega}$-hydroxy-norL-arginine or S-(2-boronoethyl)-L-cysteine restored flow-induced responses in arterioles from diabetic rats and abolished differences between diabetic and control animals. Similarly, acute in vitro pretreatment with L-arginine returned flow-mediated vasodilation in vessels from diabetic animals to that of control rats. In contrast, D-arginine failed to restore flow-induced dilation in arterioles isolated from diabetic animals. Administration of sodium nitroprusside resulted in a similar degree of dilation in arterioles isolated from control or diabetic rats. In conclusion, the present study identifies arginase as an essential mediator of skeletal muscle arteriolar endothelial dysfunction in diabetes. The ability of arginase to induce endothelial dysfunction in skeletal muscle arterioles may further compromise glucose utilization and facilitate the development of hypertension in diabetes.

Keywords: arginase, arginine, diabetes, endothelial dysfunction, flow-mediated vasodilation

\section{INTRODUCTION}

Diabetes is a progressive metabolic disease that is characterized by an elevation in circulating glucose related to either insulin deficiency (type 1 diabetes) or insulin resistance (type 2 diabetes). Diabetes and its associated complications are a growing concern and represent a serious worldwide public health problem. Vascular disease is the principal cause of morbidity and mortality in patients with diabetes (Kannel and McGee, 1979; Winer and Sowers, 2004). Accelerated atherosclerosis of the large arteries results in increased risk of myocardial infarction, stroke, and limb amputation while microvascular disease is a leading cause of blindness, nephropathy, and neuropathy (Beckman et al., 2002; Porta and Bandello, 2002; Goldberg, 2003; Kikkawa et al., 2003; Duby et al., 2004). Abnormal endothelial function is a salient feature of vascular disease in diabetes that is exemplified by a decrease in nitric oxide (NO) synthesis or bioavailability. In response to shear stress or receptor stimulation, $\mathrm{NO}$ is produced by endothelial NO synthase (eNOS) through the oxidation of its substrate, L-arginine. The release of NO by endothelial cells plays a critical role in preserving vascular homeostasis by inhibiting vascular tone, platelet aggregation, leukocyte recruitment and infiltration into the vessel wall, and smooth muscle cell proliferation and migration (see Loscalzo and Welch, 1995; Forstermann and Sessa, 2012). Endothelial dysfunction, including blunted NO-dependent vasodilatory responses, has been documented in patients and animals with diabetes, and is believed to be an important contributor to the pathogenesis of diabetic vascular disease (Durante et al., 1988; Hattori et al., 1991; Tesfamariam and Cohen, 1992; Johnson et al., 1993; Nitenberg et al., 1993). 
Although many factors have been implicated in triggering endothelial malfunction, recent studies have identified arginase as a novel mediator of endothelial dysfunction. Arginase is a metalloenzyme that hydrolyzes L-arginine to urea and L-ornithine. There are two distinct isoforms of arginase, arginase I and II, which are encoded by separate genes and share approximately $60 \%$ sequence homology (Dizikes et al., 1986; Vockley et al., 1996). Both isoforms of arginase are expressed in the vasculature but their expression is both vessel- and species-dependent (see Durante et al., 2007; Morris, 2009). Arginase elicits endothelial dysfunction by competing with eNOS for substrate L-arginine leading to a deficiency of L-arginine and diminished NO synthesis. Recent work from a number of laboratories has implicated arginase in provoking endothelial dysfunction in several pathological states, including arterial hypertension, pulmonary hypertension, atherosclerosis, aging, and hemorrhagic shock (Xu et al., 2004; Demougeot et al., 2005; Johnson et al., 2005, 2010; Ryoo et al., 2008; Kim et al., 2009). In a seminal study, Romero et al. (2008) demonstrated increased vascular arginase I expression and activity in streptozotocin-treated rats and that arginase contributes to endothelial dysfunction in coronary arteries in this animal model of type 1 diabetes. In addition, it was found that arginase stimulates endothelial dysfunction in myocardial microvessels in type 2 diabetic rats and in coronary arterioles isolated from patients with type 2 diabetes (Beleznai et al., 2011; Gronros et al., 2011). Subsequently, arginase was also shown to impair endothelial function in the aorta, retinal arteries, and corpora cavernosa of streptozotocininduced type 1 diabetic animals (Toque et al., 2011; El-Bassossy et al., 2012; Romero et al., 2012; Elms et al., 2013). However, the involvement of arginase in mediating endothelial dysfunction in other vascular beds of diabetic animals is not known.

A majority of the studies examining the contribution of arginase to endothelial dysfunction in diabetes utilize acetylcholine to test the vasoactive function of the endothelium. However, a physiological role for acetylcholine in the local regulation of vascular resistance has not been established. Furthermore, in many vascular beds a large portion of acetylcholine-mediated vasodilation is NO-independent (Bolz et al., 1999). Thus, the use of acetylcholine may not fully reveal the nature and physiologic importance of endothelial dysfunction in diabetes. A major in vivo stimulus for the synthesis and release of NO by endothelial cells is luminal flow which functions to continuously modulate arterial diameter via changes in shear stress. In order to more fully evaluate the role of arginase in promoting endothelial dysfunction in type 1 diabetes, we determined the expression of arginase I and II in skeletal muscle arterioles in rats treated with streptozotocin or vehicle. In addition, we examined endothelial function in these arterioles in response to a highly relevant physiologic stimulus: luminal flow. Finally, the response of these arterioles to an endothelium-independent vasodilator was also assessed.

\section{MATERIALS AND METHODS \\ MATERIALS}

L-Arginine, D-arginine, glycerol, sodium dodecyl sulfate (SDS), Triton X-100, Tris, sodium acetate, streptozotocin, sodium fluoride, heparin, and sodium nitroprusside were from Sigma-Aldrich (St. Louis, MO, USA). Aprotinin and leupeptin were from Roche
Applied Sciences (Indianapolis, IN, USA). $N^{\omega}$-hydroxy-norL-arginine (L-OHNA) and $S$-(2-boronoethyl)-L-cysteine (BEC) were purchased from EMD Biosciences (San Diego, CA, USA). [ Guanido- $\left.{ }^{14} \mathrm{C}\right]_{\mathrm{L}}$-arginine $(52 \mathrm{Ci} / \mathrm{mmol})$ was from Amersham Life Sciences (Arlington Heights, IL, USA). All other chemicals were obtained from Fisher Scientific (Houston, TX, USA). Sodium nitroprusside $(10 \mathrm{mM})$ stock solutions were prepared in saline and diluted in modified Krebs buffer immediately before use. L-OHNA $(100 \mu \mathrm{M})$ and BEC $(100 \mu \mathrm{M})$ were dissolved in Krebs buffer just before use. The composition of the modified Krebs buffer was (in $\mathrm{mM}) 118.5 \mathrm{NaCl}, 4.7 \mathrm{KCl}, 1.4 \mathrm{CaCl}_{2}, 1.2 \mathrm{KH}_{2} \mathrm{PO}_{4}, 1.1 \mathrm{MgSO}_{4}$, $25.0 \mathrm{NaHCO}_{3}$, and 11.1 dextrose.

\section{ANIMAL MODEL}

Adult male Sprague Dawley rats between 12 and 14 weeks of age were purchased from Charles River Laboratories (Wilmington, MA, USA). Diabetes was induced by a single injection of streptozotocin $(65 \mathrm{mg} / \mathrm{kg}$, ip) dissolved in sodium citrate $(50 \mathrm{mM})$. Nondiabetic, control animals were injected with an equivalent volume of vehicle. Animals were fed standard rat chow, had free access to drinking water, and were used four weeks after streptozotocin or vehicle administration. All experiments conform to the Guide for the Care and Use of Laboratory Animals published by the National Institutes of Health (NIH Publication No. 85-23, revised 1996) and were approved by the institutional care and use committee.

\section{HEMODYNAMIC AND METABOLIC MEASUREMENTS AND TISSUE EXTRACTIONS}

Four weeks after the streptozotocin or vehicle administration, rats were weighed and injected intraperitoneally with ketamine $(100 \mathrm{mg} / \mathrm{kg})$ and zylazine $(7.5 \mathrm{mg} / \mathrm{kg}$ ) (Butler Schein Animal Health Corporation, Dublin, OH, USA), and a carotid arterial catheter implanted for blood sample collection and blood pressure measurement. Blood samples were drawn for immediate determination of blood glucose (Accu-Chek Compact, Roche Diagnostics, Indianapolis, IN, USA), glycated hemoglobin (HbA1c, DCA 2000+ Analyzer, Bayer, Pittsburgh, PA, USA), and cholesterol (CardioChek PA Analyzer, Polymer Technology Systems, Inc., Indianapolis, IN, USA). Blood pressure was measured using a pressure transducer (TSD 104A, Biopac Systems, Santa Barbara, CA, USA) coupled to a polygraph system (Biopac Systems, Santa Barbara, CA, USA) and a personal computer. Animals were then heparinized (1000 U/kg, iv) and the thoracic aorta and gracilis anticus muscles removed and placed into ice-cold modified Krebs buffer or frozen in liquid nitrogen and stored at $-70^{\circ} \mathrm{C}$ for later use.

\section{ARGINASE ACTIVITY}

Arginase activity was determined by monitoring the formation of $\left[{ }^{14} \mathrm{C}\right]$ urea from $\left[\right.$ guanido- $\left.{ }^{14} \mathrm{C}\right] \mathrm{L}$-arginine, as we previously reported (Peyton et al., 2009). Blood vessels were sonicated in Tris buffer (10 mm, pH 7.4) containing Triton X-100 (0.4\%), leupeptin $(10 \mathrm{mg} / \mathrm{mL})$, and aprotinin $(10 \mathrm{mg} / \mathrm{mL})$. Lysates $(100 \mu \mathrm{g})$ were added to an equal volume of Tris buffer $(10 \mathrm{mM}, \mathrm{pH} 7.4)$ containing $\mathrm{MnCl}_{2}(10 \mathrm{mM})$ and arginase was activated by heating for $10 \mathrm{~min}$ at $56^{\circ} \mathrm{C}$. The arginase reaction was initiated by adding Tris buffer containing $\mathrm{L}$-arginine $(10 \mathrm{mM})$ and [guanido$\left.{ }^{14} \mathrm{C}\right]_{\mathrm{L}}$-arginine $(0.25 \mathrm{Ci})$, and samples were incubated at $37^{\circ} \mathrm{C}$ for 
$30 \mathrm{~min}$. Reactions were terminated by adding ice-cold sodium acetate buffer $(250 \mathrm{mM}, \mathrm{pH} 4.5)$ containing urea $(100 \mathrm{mM})$. $\left[{ }^{14} \mathrm{C}\right]$ Urea was separated from basic amino acids by Dowex chromatography and $\left[{ }^{14} \mathrm{C}\right]$ urea formation determined by scintillation counting.

\section{ARGINASE EXPRESSION}

Arginase expression was determined by quantitative real-time PCR. Total RNA was isolated from gracilis muscle arterioles using TRIzol reagent and quantified by absorbance spectroscopy. cDNA was synthesized with $1 \mu \mathrm{g}$ of RNA using iScript cDNA synthesis kits (Bio-Rad, Hercules, CA, USA). Quantitative real-time PCR was carried out using a SYBR Green Supermix (Bio-Rad, Hercules, CA, USA) and arginase primers using the Bio-Rad CFX96 system (Sasatomi et al., 2008). Thermal cycling was performed at $95^{\circ} \mathrm{C}$ for $10 \mathrm{~min}$ followed by 40 cycles at $95^{\circ} \mathrm{C}$ for $15 \mathrm{~s}$ and $60^{\circ} \mathrm{C}$ for $1 \mathrm{~min}$. Reactions were performed in triplicate. Relative expression of arginase was analyzed using the delta-delta $\mathrm{Ct}$ method and results normalized with respect to $18 \mathrm{~S}$ rRNA.

\section{ISOLATED MICROVESSEL EXPERIMENTS}

Segments of first-order gracilis muscle arterioles were isolated by microdissection and cannulated at both ends with glass micropipettes in a vessel chamber (Living Systems Instrumentation, Burlington, VT, USA). The vessel chamber was continuously perfused with Krebs buffer equilibrated with a gas mixture of $14 \%$ $\mathrm{O}_{2}$ and $5 \% \mathrm{CO}_{2}$, balanced with $\mathrm{N}_{2}$, in a non-recirculating system. For internal diameter measurements, the vessel chamber was mounted on a stage of an inverted microscope (TS 100-F, Nikon Instruments, Melville, NY, USA) fitted with a CCD video camera. The camera was connected to a personal computer equipped with video dimensioning software (ImagePro Express, Media Cybernetics, Bethesda, MD, USA). Images were collected at 1 frame/s and stored as digital files for documentation.

To study agonist-induced dilation, the proximal micropipette was connected to a pressure servo controller (Living Systems Instrumentation, Burlington, VT, USA) and the distal micropipette was connected to a closed stopcock to achieve $80 \mathrm{mmHg}$ constant luminal pressure with no flow. Following a $60 \mathrm{~min}$ stabilization period, peak responses of arterioles to cumulative additions of sodium nitroprusside (10-1000 $\mathrm{nM}$ ) were determined by adding the drug to the superfusion buffer. To study flow-induced dilation, both the proximal and distal micropipettes were connected to pressure servo controllers and to an inline micro flowmeter (Living Systems Instrumentation, Burlington, VT, USA). During a $60 \mathrm{~min}$ stabilization period, both proximal and distal pressures were adjusted to $80 \mathrm{mmHg}$ with no luminal flow. In order to establish graded levels of luminal flow $(0-50 \mu \mathrm{L})$, proximal and distal pressures were adjusted equally in the opposite direction maintaining midline pressure at $80 \mathrm{mmHg}$. In some experiments, arginase inhibitors or arginine was added to the superfusion buffer $20 \mathrm{~min}$ before arterioles were exposed to luminal flow. In order to limit the possible loss of vascular $\mathrm{L}$-arginine from vessels perfused with buffer devoid of L-arginine that may potentially mask differences between the two groups of animals, the duration of experiments was restricted to a single flow-response curve per vessel.

\section{STATISTICS}

Results are expressed as mean \pm SEM. Statistical analyses were performed with the use of a Student's two-tailed $t$-test and an analysis of variance with the Tukey post hoc test when more than two treatment regimens were compared. $p$-Values $<0.05$ were considered statistically significant.

\section{RESULTS}

Four weeks after administration of streptozotocin, rats develop overt diabetes as reflected by elevated fasting blood glucose and glycated hemoglobin levels compared to vehicle-treated control animals (Table 1). In addition, body weights of streptozotocindiabetic rats were significantly lower than control animals. However, there was no significant difference in blood pressure, heart rate, or circulating cholesterol levels between control and diabetic rats. Vascular arginase activity was markedly increased in diabetic rats by nearly twofold (Figure 1A). The rise in aortic arginase activity in diabetic animals was associated with a significant increase in the expression of both arginase I and II mRNA in gracilis muscle arterioles (Figure 1B).

Endothelial function was examined in isolated skeletal muscle arterioles. Increases in luminal flow $(0-50 \mu \mathrm{L} / \mathrm{min})$ resulted in progressive vasodilation in arterioles isolated from control rats (Figure 2). However, flow-mediated vasodilation was absent in arterioles isolated from diabetic animals. In fact, a

Table 1 | Metabolic and hemodynamic parameters of control and diabetic rats.

\begin{tabular}{lcc}
\hline & Control & Diabetic \\
\hline Body weight $(\mathrm{g})$ & $472 \pm 10$ & $288 \pm 14^{*}$ \\
Blood pressure (mmHg) & $122 \pm 9$ & $114 \pm 12$ \\
Heart rate (beats/min) & $328 \pm 22$ & $302 \pm 15$ \\
Glucose (mg/dL) & $98 \pm 8$ & $411 \pm 32^{*}$ \\
Glycated hemoglobin (\%) & $3.9 \pm 0.1$ & $7.9 \pm 0.2^{*}$ \\
Cholesterol (mg/dL) & $78 \pm 6$ & $87 \pm 11$
\end{tabular}

Results are means \pm SEM $(n=14-17)$.

* Statistically significant effect of diabetes $(p<0.01)$.

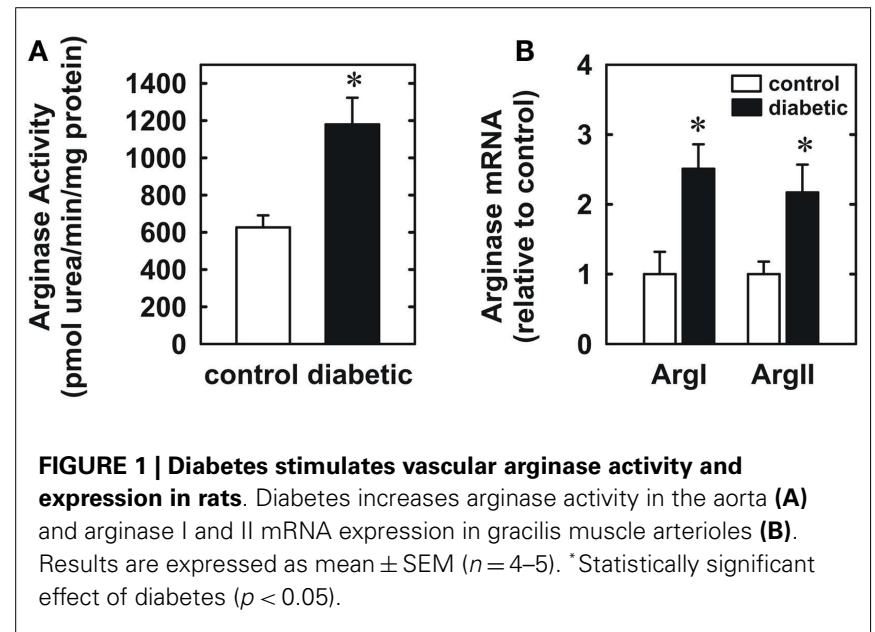




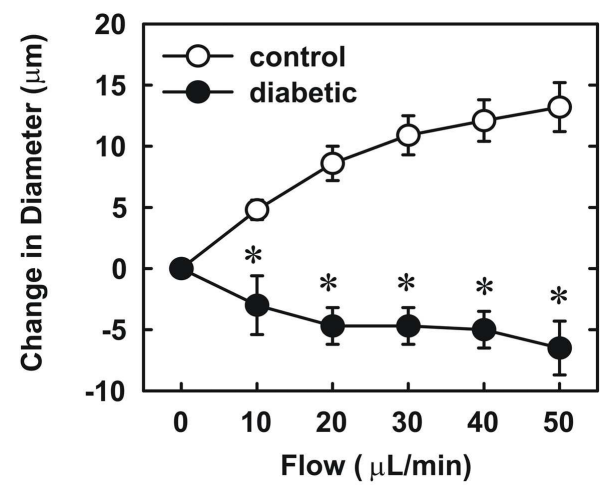

FIGURE 2 | Diabetes abolishes flow-dependent increases in internal diameter of gracilis muscle arterioles. Arterioles were isolated from control (open circles) or diabetic (closed circles) rats and exposed to graded levels of luminal flow. Results are expressed as mean \pm SEM $(n=3-5)$. ${ }^{*}$ Statistically significant effect of diabetes $(p<0.01)$.

slight vasoconstrictor effect was noted in diabetic vessels subjected to luminal flow. Since arginase expression was elevated in gracilis muscle arterioles of diabetic rats, we examined if arginase was responsible for impairing endothelial function in these animals. Acute in vitro pretreatment of blood vessels with the arginase inhibitors, L-OHNA $(100 \mu \mathrm{M})$ or BEC $(100 \mu \mathrm{M})$, restored flow-induced responses in arterioles from diabetic animals and abolished differences between the two groups of animals (Figures 3A,B). Similarly, acute in vitro pretreatment of vessels with the arginase and NO synthase substrate, L-arginine $(1 \mathrm{mM})$, reinstated flow-induced dilation in arterioles obtained from diabetic rats and abrogated the difference between control and diabetic animals (Figure 4A). In contrast, D-arginine ( $1 \mathrm{mM}$ ), which is not a substrate for either enzyme, failed to restore flowmediated responses in arterioles from diabetic rats (Figure 4B). Finally, the responsiveness of arteriole smooth muscle to NO was tested by treating vessels with the NO donor, sodium nitroprusside. Sodium nitroprusside dilated arterioles isolated from control or diabetic animals in a similar concentration-dependent manner (Figure 5).

\section{DISCUSSION}

In the present study, we found that vascular arginase activity is elevated in streptozotocin-treated diabetic rats and this is associated with a significant increase in the expression of arginase I and II in gracilis muscle arterioles. In addition, we discovered that luminal flow-induced vasodilation is severely compromised in gracilis muscle arterioles isolated from diabetic rats while the vasodilator response to the endothelium-independent agonist, sodium nitroprusside, is preserved in these vessels. We also found that acute in vitro pretreatment with arginase inhibitors restores flowinduced vasodilation and abolishes the difference between control and diabetic arterioles. Similarly, acute in vitro pretreatment with the arginase and eNOS substrate, $\mathrm{L}$-arginine, but not the inactive $\mathrm{D}$ isomer, restores flow-mediated vasodilation and eliminates the difference between the two groups of animals. These findings suggest that increased arginase activity contributes to skeletal muscle
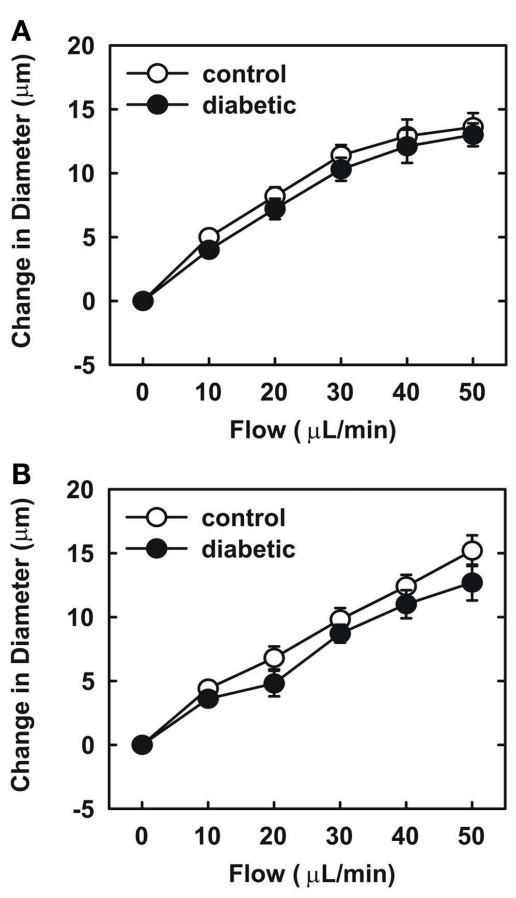

FIGURE 3 | Arginase inhibition restores flow-dependent increases in internal diameter of gracilis muscle arterioles in diabetes. Arterioles isolated from control (open circles) or diabetic (closed circles) rats were treated in vitro with the arginase inhibitors nor-NOHA (100 $\mu \mathrm{M})$ (A) or BEC $(100 \mu \mathrm{M})(\mathbf{B})$ and then exposed to graded levels of luminal flow. Results are expressed as mean $\pm \operatorname{SEM}(n=5)$.

arteriolar endothelial dysfunction in type 1 diabetes by restricting the availability of L-arginine.

Diabetes was induced in our study by a single injection of streptozotocin. This glucose moiety selectively destroys the insulin-producing $\beta$-cells of the pancreas leading to rapid insulindeficiency and diabetes. This animal model displays polydipsia, polyuria, hyperglycemia, and weight loss that are similar to the clinical symptoms found in diabetic patients (Wei et al., 2003; Akbarzadeh et al., 2007). In agreement with this, we found that rats treated with streptozotocin develop frank diabetes 4 weeks after treatment as indicated by raised fasting blood glucose and glycated hemoglobin concentrations and loss in body weight. The significant decline in body weight is more pronounced in adult animals in this model and likely reflects the loss of glucose in the urine and an inability to metabolize carbohydrates and a shift to fat metabolism leading to depletion of fat stores (Hoybergs et al., 2008).

Our finding that vascular arginase activity is increased in type 1 diabetic rats is consistent with recent studies in streptozotocintreated rats and mice where elevated arginase activity was reported in the liver, aorta, kidney, macrophages, retina, and corpora cavernosa (Romero et al., 2008, 2012; Morris et al., 2011; Toque et al., 2011; Sun et al., 2012; Elms et al., 2013). For the first time, we also show that diabetes induces the expression of both arginase I and II in gracilis muscle arterioles. This novel observation contrasts with earlier studies showing that diabetes selectively stimulates 

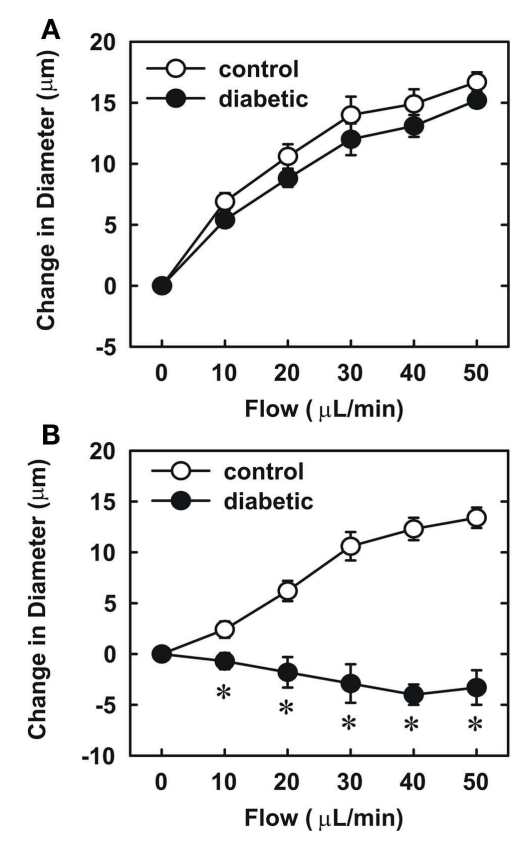

FIGURE 4 | Effect of arginine on flow-dependent increases in internal diameter of gracilis muscle arterioles. Arterioles isolated from control (open symbol) or diabetic (closed symbol) rats were treated in vitro with the L-arginine (1 $\mathrm{mM}) \mathbf{( A )}$ or D-arginine $(1 \mathrm{mM}) \mathbf{( B )}$ and then exposed to graded levels of luminal flow. Results are expressed as mean \pm SEM $(n=4-5)$. *Statistically significant effect of diabetes $(p<0.01)$.

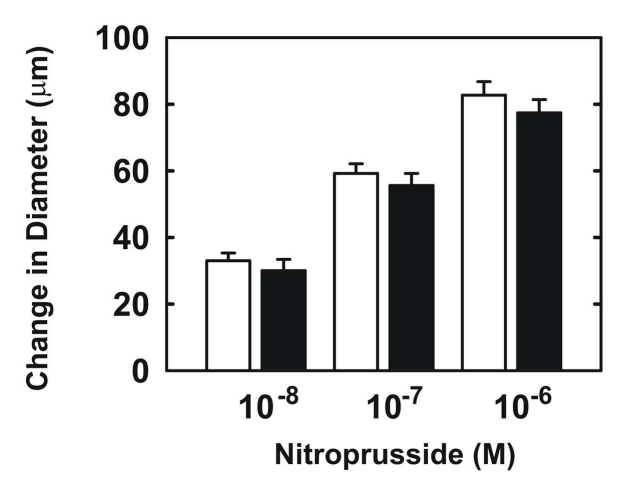

FIGURE 5 | Sodium nitroprusside-mediated increases in internal diameter of gracilis muscle arterioles. Arterioles isolated from control (open symbol) or diabetic (closed symbol) rats were exposed to various concentrations of sodium nitroprusside. Results are expressed as mean $\pm \operatorname{SEM}(n=5)$.

arginase I or II expression in different tissues, further underscoring the tissue and vessel-dependent pattern of arginase expression (see Durante et al., 2006; Morris, 2009). Interestingly, we previously demonstrated that arginase I and II are also both elevated in rat gracilis muscle arterioles following the development of saltsensitive hypertension, suggesting that arginase I and II are regulated in a coordinate fashion in these vessels (Johnson et al., 2005). The induction of arginase in diabetes may occur via multiple mechanisms. Since insulin was recently demonstrated to suppress plasma arginase activity in diabetic patients, the reduction or lack of circulating insulin may contribute to the increase in arginase activity in type 1 diabetes (Kashyap et al., 2008). However, hyperglycemia also appears to be involved in the widespread induction of arginase observed in diabetes as exposure of vascular cells to high concentrations of glucose triggers a rise in arginase activity (Durante et al., 2006; Romero et al., 2008). The ability of hyperglycemia to augment arginase activity likely occurs via the RhoA pathway, which can stimulate the induction or activation of both arginase isoforms (Ming et al., 2004; Romero et al., 2008).

We also found that endothelial function is markedly impaired in skeletal muscle arterioles from diabetic rats. Endothelial function was examined using a physiologically relevant stimulus, fluid flow, in first-order gracilis muscle arterioles. We previously reported that flow-mediated vasodilation in gracilis muscle arterioles from normoglycemic rats is completely abolished by eNOS inhibition, indicating that NO is responsible for flow-induced vasodilation in these vessels (Johnson and Johnson, 2008). While arterioles from control rats exhibit pronounced vasodilation in response to luminal flow, flow-induced vasodilation is absent in arterioles isolated from diabetic animals, suggesting that endothelial NO synthesis or bioavailability is dramatically reduced in these vessels. Arterioles from diabetic rats respond as well as arterioles from control animals to the NO donor, sodium nitroprusside, indicating that the sensitivity of arteriolar smooth muscle to NO is unchanged in diabetic animals. Our finding that gracilis muscle arterioles from streptozotocin-treated diabetic rats display a modest vasoconstrictor response to flow has also been reported in mesenteric arteries from these animals and may be linked to diminished NO production (Tribe et al., 1998). To test whether arginase contributes to impaired endothelial function in type 1 diabetes, we employed two distinct arginase inhibitors, L-OHNA and BEC, which are highly potent inhibitors of arginase I and II (Christianson, 2005). We found that acute pretreatment with either L-OHNA or BEC restores flow-induced dilation in arterioles from diabetic rats to levels seen in vessels from control animals. Taken in conjunction with our findings that vascular arginase I and II expression are increased in diabetic arterioles, these results suggest that arginase contributes to gracilis muscle arteriolar NO dysfunction in type 1 diabetes. Our current experimental findings build on previous work in the vasculature of the heart, retina, and corpora cavernosa (Romero et al., 2008, 2012; Toque et al., 2011) and are also supported by a recent clinical study showing arginase inhibition markedly improves endothelium-dependent vasodilation in the forearm of patients with type 2 diabetes and coronary artery disease (Shemyakin et al., 2012). Thus, arginase-mediated endothelial dysfunction may be a characteristic feature of diabetes that encompasses many vascular beds.

Our finding that arginase inhibition restores endotheliumdependent vasodilation of skeletal muscle arterioles from diabetic animals suggests that arginase may modulate L-arginine availability for NO synthesis in diabetes. Consideration of the enzyme kinetics for arginase and eNOS, indicates that arginase can effectively compete with eNOS for substrate L-arginine (Wu and Morris, 1998). Consistent with a role for arginase in depleting substrate for eNOS, vascular L-arginine concentrations 
are significantly reduced in streptozotocin-treated diabetic rats (Pieper and Dondlinger, 1997). Moreover, we showed that acute administration of L-arginine mimics the effect of arginase inhibition. L-Arginine restores flow-induced vasodilation in arterioles from diabetic rats whereas the inactive isomer, $\mathrm{D}$-arginine, has no effect. The induction of vascular arginase and the subsequent depletion of L-arginine may also explain the ability of exogenously administered $\mathrm{L}$-arginine to restore endothelial function and $\mathrm{NO}$ synthesis in both chemical and genetic animal models of type 1 diabetes (Pieper and Dondlinger, 1997; Pieper et al., 1997; Kohli et al., 2004). Furthermore, arginase-mediated decreases in intracellular L-arginine may negatively impact endothelial function by sensitizing endothelial cells to the endogenous eNOS inhibitor, asymmetric dimethylarginine, which is elevated in streptozotocin-treated rats (Lin et al., 2002). Finally, arginase-mediated depletion of arginine may further compromise endothelial function in diabetes by uncoupling eNOS (Kim et al., 2009).

The ability of arginase to inhibit endothelial function in skeletal muscle arterioles in diabetes is of pathological significance. Skeletal muscle arterioles contribute greatly to peripheral resistance and consequently are major determinants of blood pressure. Thus, arginase-mediated impairment of skeletal muscle arteriolar function may contribute to the development of hypertension in diabetes. Although blood pressure not increased four weeks after streptozotocin administration in our study, a longer duration of diabetes is associated with the development of hypertension in this animal model. Recently, El-Bassossy et al. (2012) reported that diabetic rats develop a significant increase in systolic and diastolic blood pressure 8 weeks following streptozotocin treatment. Notably, administration of arginase inhibitors for the last 6 weeks significantly reduced the developed elevation in diastolic blood pressure in these animals, illustrating a role for arginase

\section{REFERENCES}

Akbarzadeh, A., Norouzian, D., Mehrabi, M. R., Jamshidi, S., Farhangi, A., Allah Verdi, A., et al. (2007). Induction of diabetes by streptozotocin in rats. Indian J. Clin. Biochem. 22, 60-64.

Beckman, J. A., Creager, M. A., and Libby, P. (2002). Diabetes and atherosclerosis: epidemiology, pathophysiology, and management. JAMA 286, 2570-2581.

Beleznai, T., Feher, A., Spielvogel, D., Lansman, S. L., and Bagi, Z. (2011). Arginase I contributes to diminished coronary arteriolar dilation in patients with diabetes. Am. J. Physiol. Heart Circ. Physiol. 300, H777H783.

Bolz, S. S., de Wit, C., and Pohl, U. (1999). Endothelium-derived hyperpolarizing factor but not NO reduces smooth muscle $\mathrm{Ca} 2+$ during acetylcholine-induced dilation of microvessels. Br. J. Pharmacol. 128, 124-134.

Christianson, D. W. (2005). Arginase: structure, mechanism, and physiological role in male and female

in diabetes-associated hypertension. Since skeletal muscle represents a major site of insulin-dependent glucose uptake and utilization, arginase-mediated endothelial dysfunction may limit skeletal muscle perfusion and exacerbate hyperglycemia in diabetes. In support of this proposal, arginase inhibition reduces the rise in serum glucose and advanced glycation end products in type 1 diabetic rats (El-Bassossy et al., 2012), while arginase II deletion improves glucose tolerance and insulin sensitivity in type 2 diabetic mice (Ming et al., 2012). Thus, arginasemediated endothelial dysfunction of skeletal muscle arterioles may promote the vascular and metabolic derangements observed in diabetes.

In conclusion, the present study identifies arginase as a critical mediator of skeletal muscle arteriolar endothelial dysfunction in diabetes. Flow-induced vasodilation is abolished in gracilis muscle arterioles of streptozotocin-treated rats and this is associated with an increase in arteriolar arginase I and II expression. Moreover, pretreatment of blood vessels with arginase inhibitors or L-arginine fully restores flow-induced vasodilation in arterioles from diabetic rats. These results provide novel insight into the mechanism by which skeletal muscle arteriolar function is compromised in diabetes, and establishes arginase as a potential therapeutic target in treating vascular and metabolic disorders in diabetes.

\section{ACKNOWLEDGMENTS}

This work was supported by grants from the National Institutes of Health National Heart, Lung, and Blood Institute (Grants R01-HL 059976, R01-HL074966, and R01 HL076187), the Juvenile Diabetes Research Foundation, and the American Heart Association Midwest Affiliate (0855715G) and South Central Affiliates (BGIA $0865241 \mathrm{~F})$.

stimulates vascular arginase activity. FASEB J. 20, A727, abstr. 465.5.

Durante, W., Sen, A. K., and Sunahara, F. A. (1988). Impairment of endothelial-dependent relaxation in aortae from spontaneously diabetic rats. Br. J. Pharmacol. 94, 463-468.

El-Bassossy, H. M., El-Fawal, R., and Fahmy, A. (2012). Arginase inhibition alleviates hypertension associated with diabetes: effect on endothelial dependent relaxation and NO production. Vascul. Pharmacol. 57, 194-200.

Elms, S. C., Toque, H. A., Rojas, M., $\mathrm{Xu}, \mathrm{Z}$., Caldwell, R. B., and Caldwell, R. W. (2013). The role of arginase I in diabetes-induced retinal vascular dysfunction in mouse and rat models of diabetes. Diabetologia 56, 654-662.

Forstermann, U., and Sessa, W.C. (2012). Nitric oxide synthases: regulation and function. Eur. Heart J. 33, 829-837.

Goldberg, R. B. (2003). Cardiovascular disease in patients who have diabetes. Cardiol. Clin. 21, 399-413.

Gronros, J., Jung, C., Lundberg, J. O., Cerrato, R., Ostenson, C. G., and Pernow, J. (2011). Arginase inhibition restores in vivo coronary microvascular function in type 2 diabetic rats. Am. J. Physiol. Heart Circ. Physiol. 300, H1174-H1181.

Hattori, Y., Kawasaki, H., Abe, K., and Kanno, M. (1991). Superoxide dismutase recovers altered endothelium-dependent relaxation in diabetic rat aorta. Am. J. Physiol. 261, H1086-H1094.

Hoybergs, Y. M. J. J., Bierman, R. L. V., and Meert, T. F. (2008). The impact of body weight and body condition on behavioral testing for painful diabetic neuropathy in the streptozotocin rat model. Neurosci. Lett. 436, 13-18.

Johnson, F. K., Johnson, R. A., Peyton, K. J., and Durante, W. (2005) Arginase inhibition restores arteriolar endothelial function in Dahl rats with salt-induced hypertension. Am. J. Physiol. Regul. Integr. Comp. Physiol. 288, R1057-1062. 
Johnson, F. K., and Johnson, R. A. (2008). Heme oxygenase-derived endogenous carbon monoxide impairs flow-induced dilation in resistance vessels. Shock 29, 526-530.

Johnson, M. T., Creager, S. J., Scales, K. M., Cusco, J. A., Lee, B. K., and Creager, M. A. (1993). Impaired endothelium-dependent vasodilation in patients with insulindependent diabetes mellitus. Circulation 88, 2510-2516.

Johnson, R. A., Durante, W., Craig, T., Peyton, K. J., Myers, J. G., Stewart, R. M., et al. (2010). Vascular arginase contributes to arteriolar endothelial dysfunction in a rat model of hemorrhagic shock. J. Trauma 69, 384-391.

Kannel, W. B., and McGee, D. L. (1979). Diabetes and cardiovascular disease: the Framingham study. JAMA 241, 2035-2038.

Kashyap, S. R., Lara, A., Zhang, R., Park, Y. M., and Defronzo, R. A. (2008). Insulin reduces plasma arginase activity in type 2 diabetic patients. Diabetes Care 31, 134-139.

Kikkawa, R., Koya, D., and Haneda, M. (2003). Progression of diabetic nephropathy. Am. J. Kidney Dis. 41, S19-S21.

Kim, J. H., Bugai, L. J., Oh, Y. J., Bivalacqua, T. J., Ryoo, S., Soucy, K. G., et al. (2009). Arginase inhibition restores NOS coupling and reverses endothelial dysfunction and vascular stiffness in old rats. J. Appl. Physiol. 107, 1249-1257.

Kohli, R., Meininger, C. J., Haynes, T. E., Yan, W., Self, J. T., and Wu, G. (2004). Dietary L-arginine supplementation enhances endothelial nitric oxide synthesis in streptozotocin-induced diabetic rats. J. Nutr. 134, 600-608.

Lin, K. Y., Ito, A., Asagami, T., Tsao, P. S., Adimoolam, S., Kimoto, M., et al. (2002). Impaired nitric oxide synthase pathway in diabetes mellitus: role of asymmetric dimethylarginine and dimethylarginine dimethylaminohydrolase. Circulation 106, 987-992.

Loscalzo, J., and Welch, G. (1995). Nitric oxide and its role in the cardiovascular system. Prog. Cardiovasc. Dis. 38, $87-104$.
Ming, X.-F., Barandier, C., Viswambharan, H., Kwak, B. R., Mach, F., Mazzolai, L., et al. (2004). Thrombin stimulates human endothelial arginase enzymatic activity via the RhoA/ROCK pathway: implications for atherosclerotic endothelial dysfunction. Circulation 110, 3708-3714.

Ming, X.-F., Rajapakse, A. G., Yepuri, G., Xiong, Y., Carvas, J. M., Ruffieux, J., et al. (2012). Arginase II promotes macrophage inflammatory responses through mitochondrial reactive oxygen species, contributing to insulin resistance and atherogenesis. J. Am. Heart Assoc. 1, e000992.

Morris, S. M. Jr. (2009). Recent advances in arginine metabolism: roles and regulation of the arginases. $\mathrm{Br}$. J. Pharmacol. 157, 922-930.

Morris, S. M. Jr., Gao, T., Cooper, T. K., Kepka-Lenhart, D., and Awad, A. S. (2011). Arginase-2 mediates diabetic renal injury. Diabetes 60, 3015-3022.

Nitenberg, A., Valensi, P., Sachs, R., Dali, M., Aptecar, E., and Attali, J. (1993). Impairment of coronary vascular reserve and acetylcholineinduced coronary vasodilation in diabetic patients with angiographically normal coronary arteries and normal left ventricular systolic function. Diabetes 42, 1017-1025.

Peyton, K. J., Ensenat, D., Azam, M. A., Keswani, A. N., Kannan, S., Liu, X.-M., et al. (2009). Arginase promotes neointima formation in rat injured carotid arteries. Arterioscler. Thromb. Vasc. Biol. 29, 488-494.

Pieper, G. M., and Dondlinger, L. A. (1997). Plasma and vascular tissue arginine are decreased by diabetes: acute arginine supplementation restores endothelium-dependent relaxation by augmenting cGMP production. J. Pharmacol. Exp. Ther. 283, 684-691.

Pieper, G. M., Siebeneich, W., MooreHilton, G., and Roza, A. M. (1997). Reversal by L-arginine of a dysfunctional arginine/nitric oxide pathway in the endothelium of the genetic diabetic BB rat. Diabetologia 40, 910-915.
Porta, M., and Bandello, F. (2002). Diabetic retinopathy: a clinical update. Diabetologia 45, 1617-1634.

Romero, M. J., Iddings, J. A., Platt, D. H., Ali, M. I., Cederbaum, S. D., Stepp, D. W., et al. (2012). Diabetes-induced vascular dysfunction involves arginase I. Am. J. Physiol. Heart Circ. Physiol. 302, H159-H166.

Romero, M. J., Platt, D. H., Tawfik, H. E., Labazi, M., El-Remessy, A. B., Bartoli, M., et al. (2008). Diabetes-induced coronary vascular dysfunction involves increased arginase activity. Circ. Res. 102, 95-102.

Ryoo, S., Gupta, G., Benjo, A., Lim, H. K., Camara, A., Sikka, G., et al. (2008). Endothelial arginase II. A novel target for the treatment of atherosclerosis. Circ. Res. 102, 923-932.

Sasatomi, K., Hiragata, S., Miyazato, M., Chancellor, M. B., Morris, S. M. Jr., and Yoshimura, N. (2008). Nitric oxide-mediated suppression of detrusor overactivity by arginase inhibitor in rats with chronic spinal cord injury. Urology 72, 696-700.

Shemyakin, A., Kovamees, O., Rafnsson, A., Bohm, F., Svenarud, P., Settergren, M., et al. (2012). Arginase inhibition improves endothelial function in patients with coronary artery disease and type 2 diabetes. Circulation 126, 2943-2950.

Sun, C., Sun, L., Ma, H., Peng, J., Zhen, Y., Duan, K., et al. (2012). The phenotype and functional alterations of macrophages in mice with hyperglycemia for long term. J. Cell. Physiol. 227, 1670-1679.

Tesfamariam, B., and Cohen, R. A. (1992). Free radicals mediate endothelial cell dysfunction caused by elevated glucose. Am. J. Physiol. 263, H321-H326.

Toque, H. A., Tostes, R. C., Yao, L., Xu, Z., Webb, R. C., Caldwell, R. B., et al. (2011). Arginase II deletion increases corpora cavernosa relaxation in diabetic mice. J. Sex. Med. 8, 722-733.

Tribe, R. M., Thomas, C. R., and Poston, L. (1998). Flow-induced dilation in isolated resistance arteries from control and streptozotocin-diabetic rats. Diabetologia 41, 34-39.
Vockley, J. G., Jenkinson, C. P., Shukla, H., Kern, R. M., Grody, W. W., and Cederbaum, S. D. (1996). Cloning and characterization of the human type II arginase gene. Genomics 2, 118-123.

Wei, M., Ong, L., Smith, M. T., Ross, F. B., Schmid, K., Hoey, A. J., et al. (2003). The streptozotocindiabetic rat model as a model of the chronic complications of human diabetes. Heart Lung Circ. 12, 44-50.

Winer, N., and Sowers, J. R. (2004). Epidemiology of diabetes. J. Clin. Pharmacol. 44, 397-405.

Wu, G., and Morris, S. M. Jr. (1998). Arginine metabolism: nitric oxide and beyond. Biochem. J. 336, 1-7.

$\mathrm{Xu}$, W., Kaneko, F. T., Zheng, S., Comhair, S. A., Janocha, A. J., Goggans, T., et al. (2004). Increased arginase II and decreased NO synthesis in endothelial cells of patients with pulmonary hypertension. FASEB J. 18, 1746-1748.

Conflict of Interest Statement: The authors declare that the research was conducted in the absence of any commercial or financial relationships that could be construed as a potential conflict of interest.

Received: 20 March 2013; paper pending published: 08 April 2013; accepted: 04 May 2013; published online: 20 May 2013.

Citation: Johnson FK, Johnson RA, Peyton KJ, Shebib AR and Durante W (2013) Arginase promotes skeletal muscle arteriolar endothelial dysfunction in diabetic rats. Front. Immunol. 4:119. doi: 10.3389/fimmu.2013.00119

This article was submitted to Frontiers in Inflammation, a specialty of Frontiers in Immunology.

Copyright (C) 2013 Johnson, Johnson, Peyton, Shebib and Durante. This is an open-access article distributed under the terms of the Creative Commons Attribution License, which permits use, distribution and reproduction in other forums, provided the original authors and source are credited and subject to any copyright notices concerning any third-party graphics etc. 